\title{
النموذج النظري لقرار التهرب من ضريبة الدخل
}

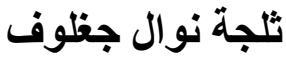 كلية العلوم الاقتصـادية \\ جامعة عبد الحمبد مهري الافئمي قسنطينة 2}

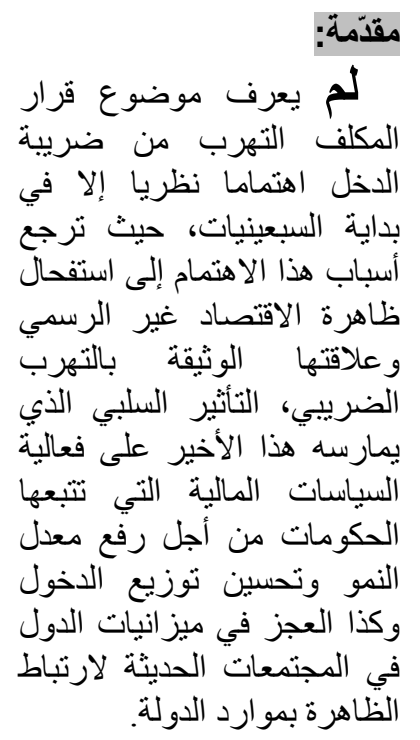

قدمت، منذ السبعينيات، العديد من النماذج النظرية لتحديد

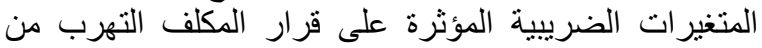

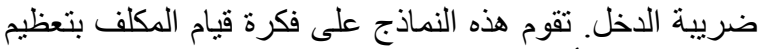

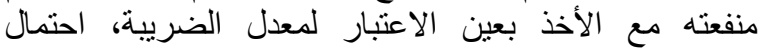

الاكتشاف نتيجة الخضوع للمراقبة وكذا مدى ردعية عقوبة الإن التهرب الضريبي.

سنحاول في هذه المقالة التركيز على دور المتغير الضريبيفي

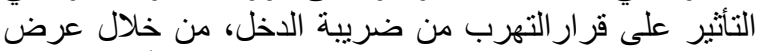

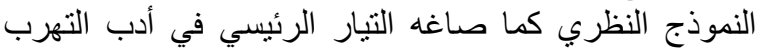
الضريبي. الكلمـات المفتاحية: الضريبة على الاخل، قرار التهرب من التربه ضريبة الدخل، نموذج ألينغهاموساندمو

\section{Résumé :}

Depuis les années soixante-dix, des modèles théoriques ont été avancés pour déterminer l'influence des variables fiscales sur la décision de l'évasion de l'impôt sur le revenu. Ces modèles supposent que le contribuable cherche à maximiser son utilité en tenant compte du taux d'imposition, de la probabilité de détection ainsi que de la pénalité pour évasion fiscale. Nous essayons dans cet article, de déterminer dans quelle mesure la variable fiscale influence la décision de l'évasion de l'impôt sur le revenu, à travers la présentation du modèle conceptuel tel que formulé par le courant dominant dans la littérature d'évasion fiscale.

Mots-clés : impôt sur le revenu, la décision de l'évasion de l'impôt sur le revenu, modèle ALLINGHAM et SANDMO 


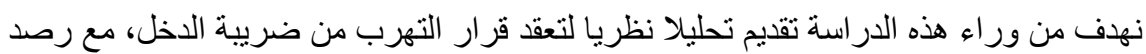

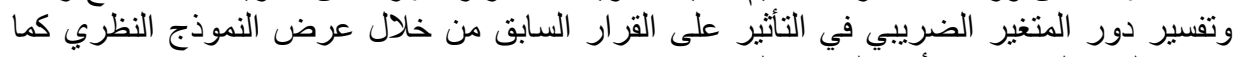

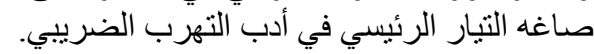

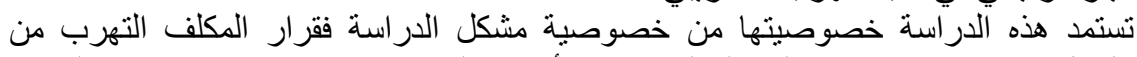

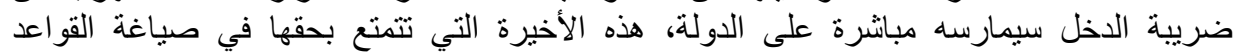

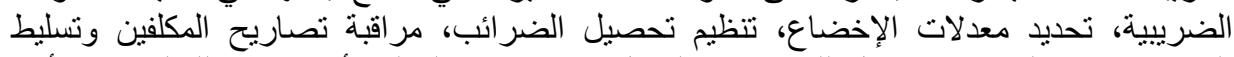

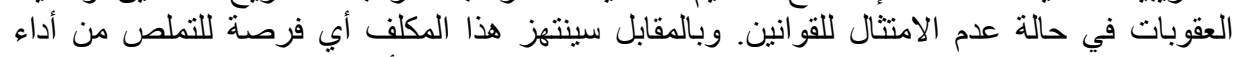

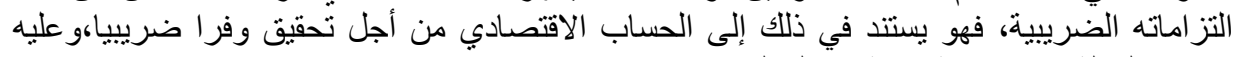

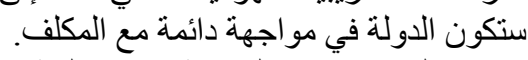
لقد اعتمدت الدراسة في معالجة إنكالية الموضوع علد على المنهج الوصفي التحليلي والمنهج الرياضي من خلال التعرض للعناصر التالية:

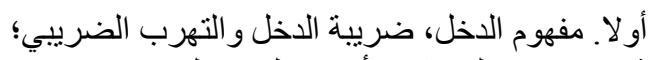

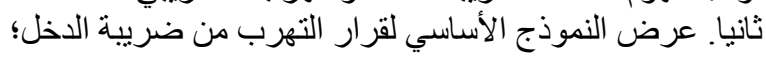

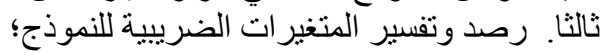

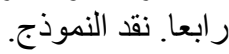

\section{أولا. مفهوم الدخل، ضريبة الدخل و التهرب الضريبي}

لم يرد تعريف واضح للاخل، لكن المتداول أن الدخل هو ما يحصل عليه الثخص من أموال

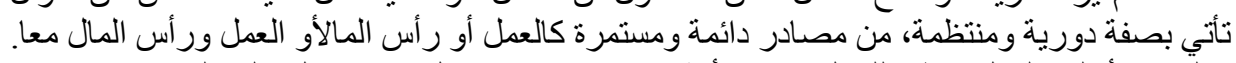

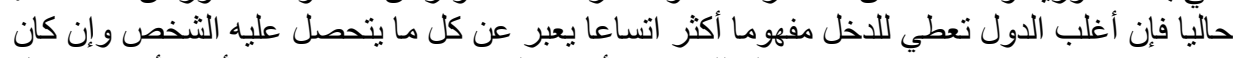

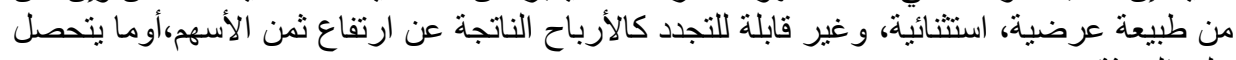

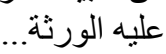

و وعليه يكفي مقارنة ذمة الثخص في أول السنة وذمته في أخر السنة، وذلك لاكتشاف أي زيادة حاصلة

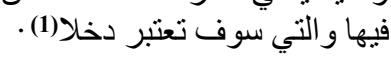

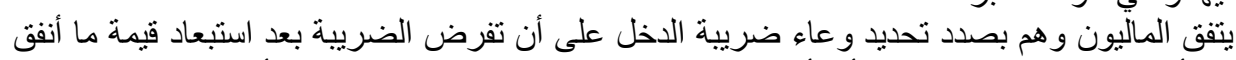

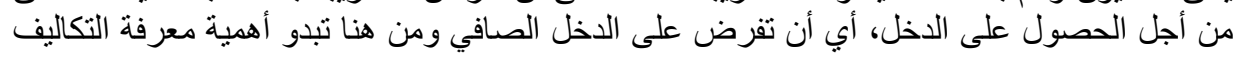

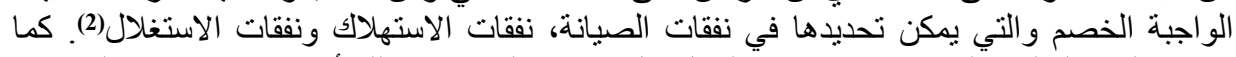

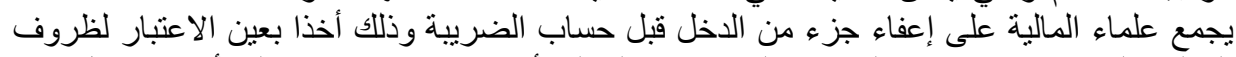

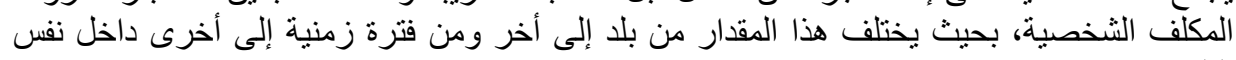

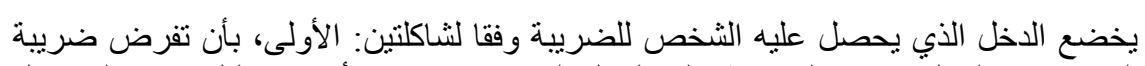
البلا.

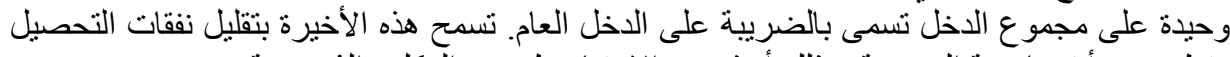

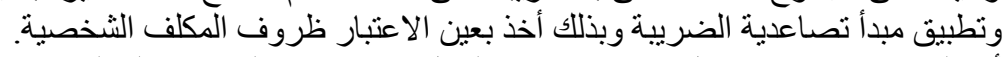

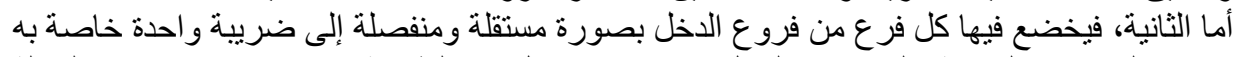

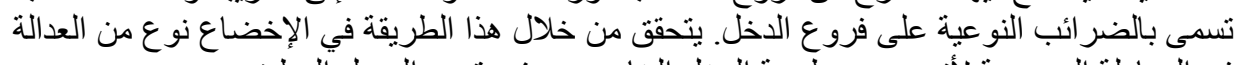

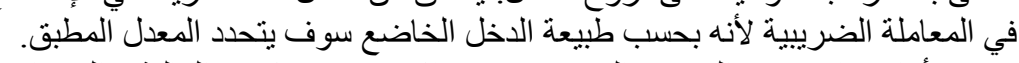

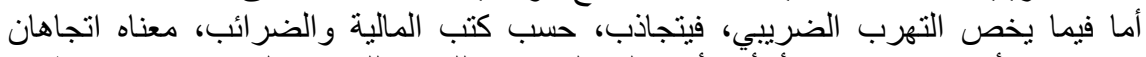

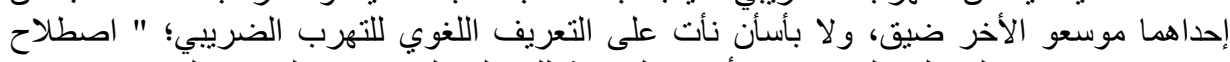

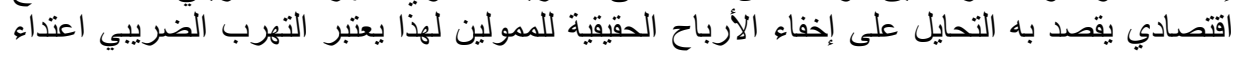


على حقوق الخزانة العامة"(3)،ونجد في معجم لسان العرب تحت كلمة هرب: فروهرب الإنسان أي

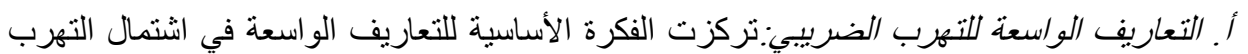

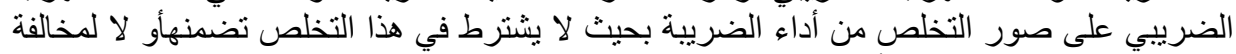
للقانونوذلك على النحو المبين أدناه:

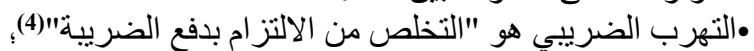

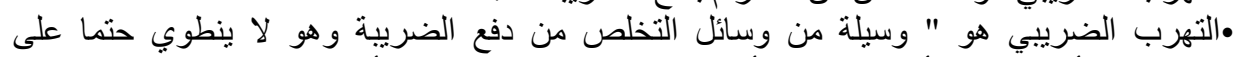

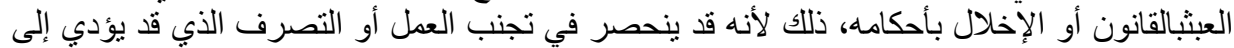

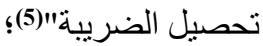
•التهرب الضريبي هو "كل وسيلة لجأ إليها الممول في سبيل التخلص من عبء ضريبي يفترض وقو عه على عاتقه" (6).

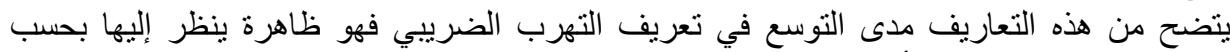

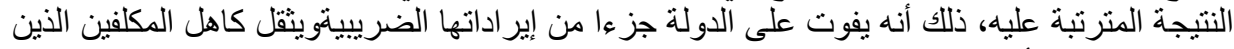

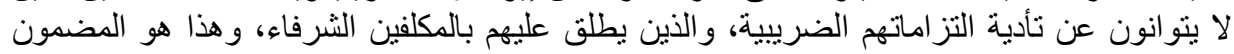

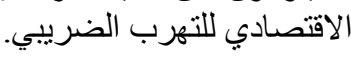

ب. التعاريف الضيقة للتهرب الضربيي:تثترط التعاريف الضيقة لوقوع التهرب الضريبي تو افر الوسيلة

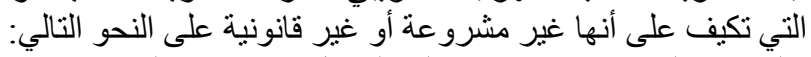

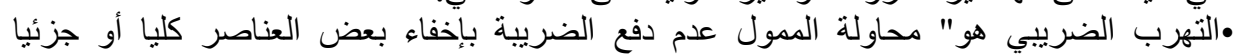

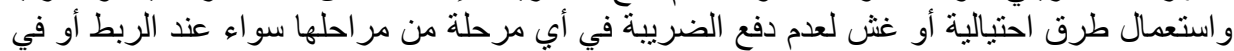

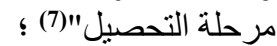

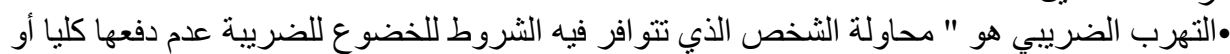

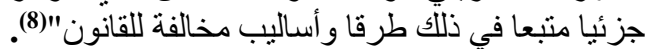

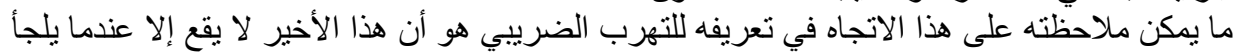

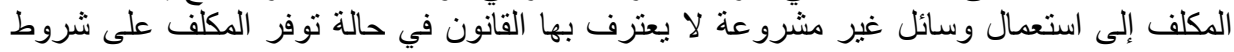

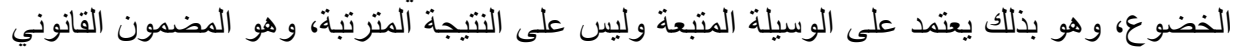
اللتهرب الضرعيبي.

تتولد الدخول المتهرب من دفع الضرائب عليها عن أنشطة غير قانونية، كالاتجار في السلع

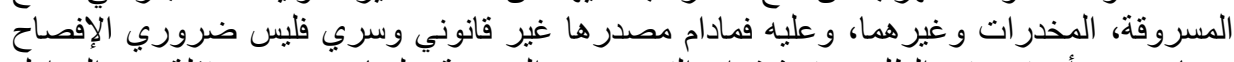

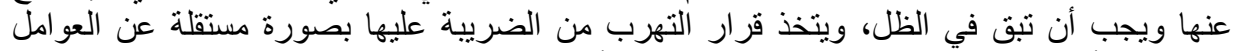

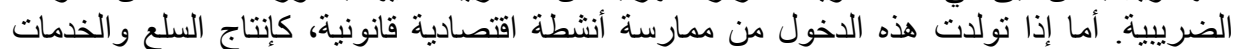

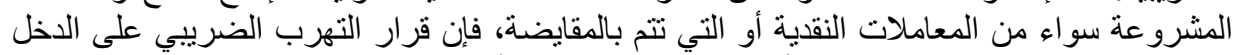

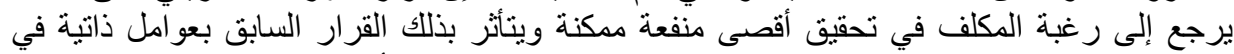

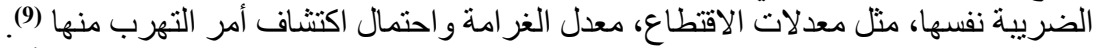

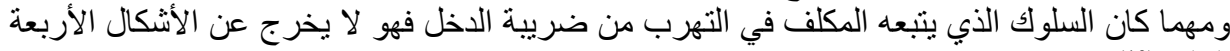

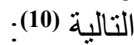

• عدم الإعلان أصلا عن النشاط وبالتالي عن الاخل الخاضع للضريبة؛

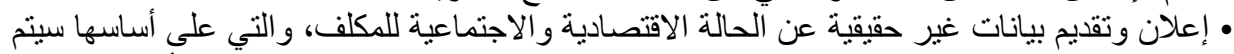

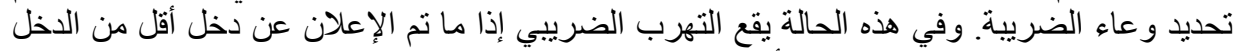

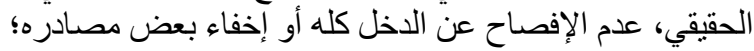




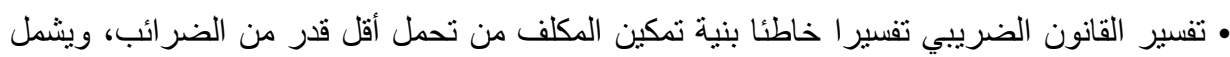

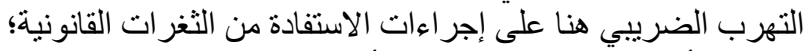
• إتباع الأساليب الثناثة السابقة كلها أو بعضها.

ثثاتيا. عرض النموذج الأساسي لقرار التهرب من ضريبة الدخل:

بدأ الاهتمام، منذ بداية السبعينيات، بالدراسة النظرية لقرار التهرب من ضريبة الدخل، والتئي تستند على التئي

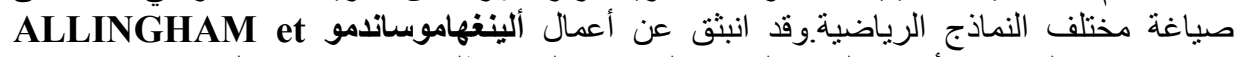
SANDMO

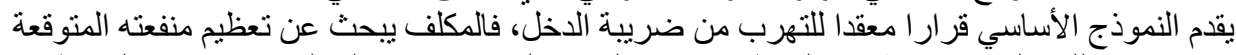

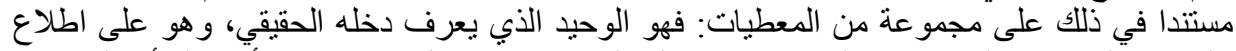

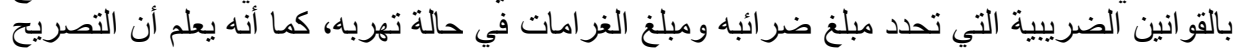

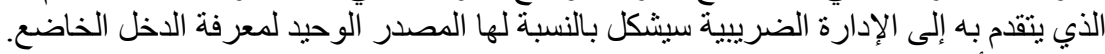

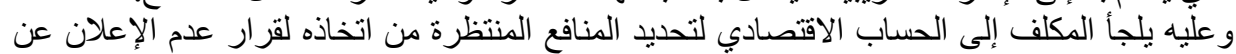

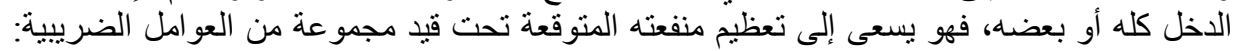

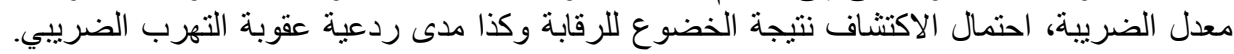

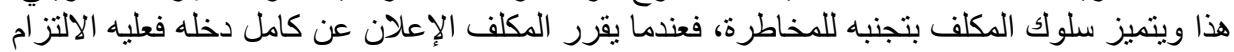

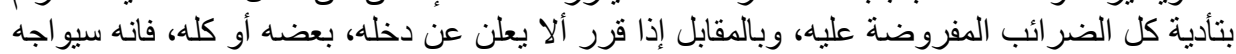

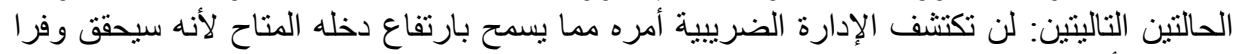

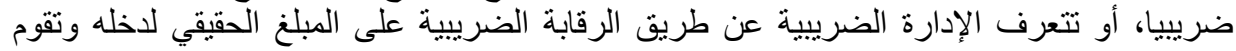

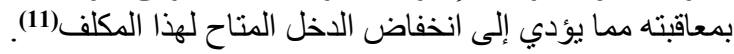

$$
\text { أ فرضيات ومعطيات النموذج }
$$

يؤكد النموذج على الفرضيات التالية(12):

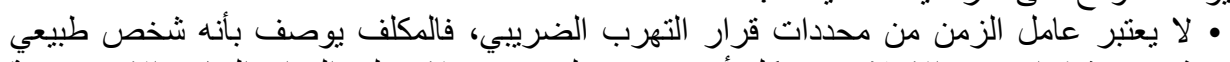
ورشيد يتخذ قرار عدم الإعلان عن كل أو بعض دخله بله، بحيث لا يتعلق القرار السابق إلا بمدة سنة

واحدة؛ ورثن

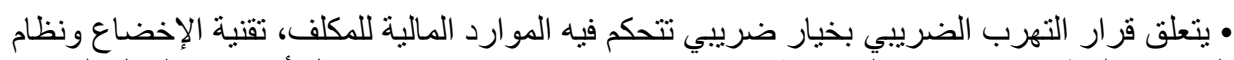

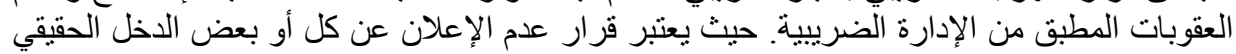

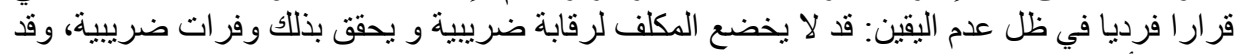

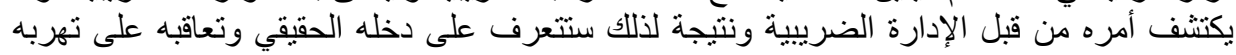
الضريبي؛

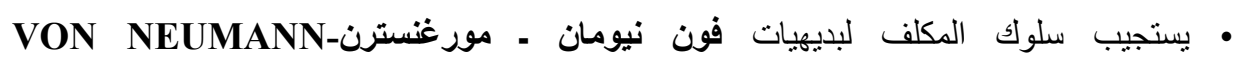
MORGRNSTERN

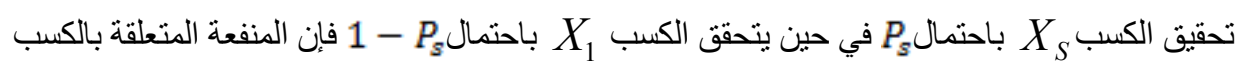

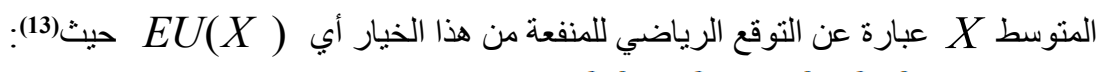
$E U(X)=\left(1-P_{S}\right) U\left(X_{1}\right)+P_{S} U\left(X_{s}\right)$ 
ومنه يختار المكلف الفعل الذبي يعظم منفعته المتوقعة بحيث تحقق دالة المنفعة لفون نيومان مورغنسترن(VON NEUMANN-MORGRNSTERN) الخصائص التالية: تكون دالة المنفعة تابعة فقط لدخل المكلف المتاح؛ ل ل ح لكون دالة المنفعة متز ايدة، أما دالة المنفعة الحدية فهي مو جبة ومتناقصة:

$$
\text { . ( } \left.U^{\prime \prime} \prec 0, U^{\prime} \succ 0\right)
$$

• يفترض أن يكون المكلف ينفر من المخاطرة وتدل المخاطرة على نوقع اختلافات في العائد بين

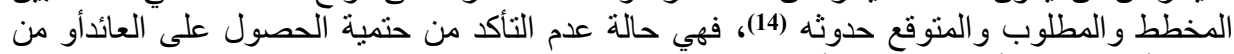

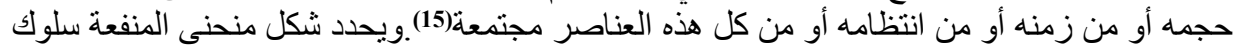

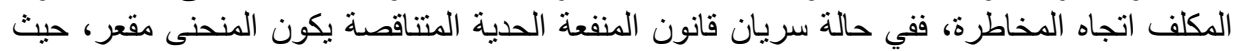

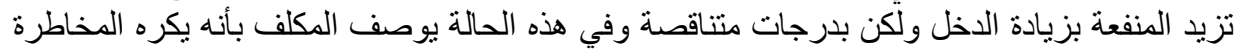

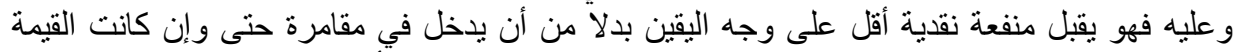

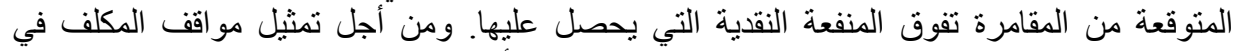

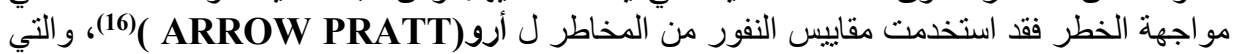
تم تعريفها وترميز ها كما يلي:

$$
\begin{aligned}
& A(k)=-\frac{U^{\prime \prime}(k)}{U^{\prime}(k)} \\
& R(k)=-\frac{U^{\prime \prime}(k) k}{U^{\prime}(k)} \\
& \text { و }
\end{aligned}
$$

وبإسقاط ما سبق تتاوله يمكننا التعريف بالرموز التالية التي استخدها النموذج:

U : دالة المنفعة والتي تترجم نفور المكلف من المخاطرة؛ : Rr

Rd $R_{n d}$

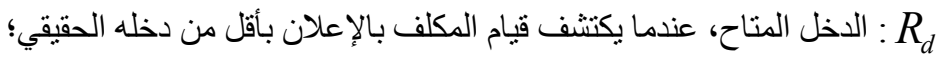

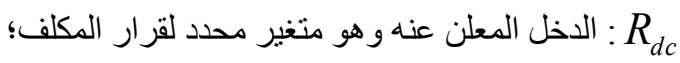
t

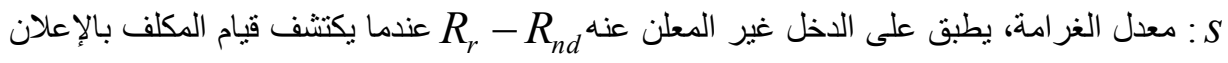
بأقل من دخله الحقيقي، حيث وغر امة اخفاء جزء من الدخل الحقيقي؛ 
p : احتمال الاكتثاف، عندما تقوم الإدارة الضريبية بالرقابة الضريبية بصفة عشو ائية وتكتثف الدخل

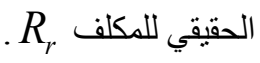

• $R_{n d}=R_{r}-t R_{d c}$ ، حيث ينجح المكلف في إخفاء جزء من دخله دون أن تكتثف الإدارة الضريبية

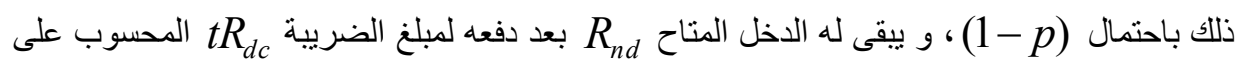
الجزء من الدخل المعلن عنه فقط $R_{d c}$ و الذي يكون أقل من الحقيقي $R_{r}$ و و عليه هناك جزء لـاء من الدخل أخفاه المكلف على الإدارة الضريبية يقدر ب

•

لخضوعه للرقابة الضريبية من قبل الإدارة الضريبية سوف نكتثف ذللك و هذا باحتمال (p) و وتتعرف

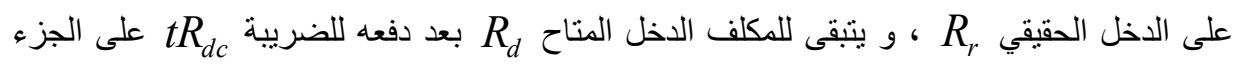

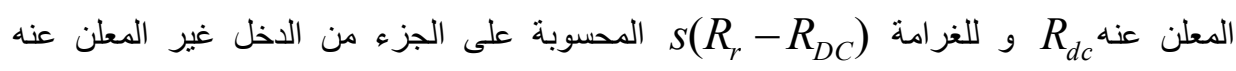

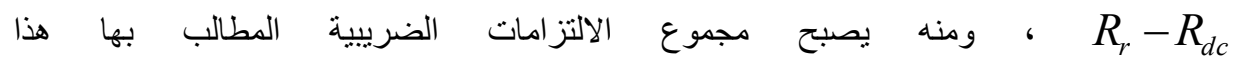
المكلف

، ع الةقالمنفعة المتوقعة في الدخل المتاح الذي يحققه

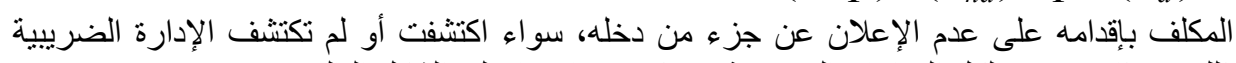

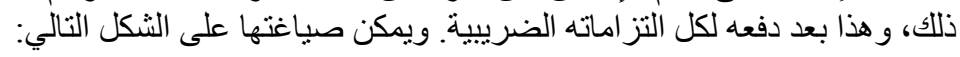

$$
E U=(1-p) U\left(R_{r}-t R_{d c}\right)+p U\left(R_{r}-t R_{d c}-s\left(R_{r}-R_{d c}\right)\right)[1]
$$

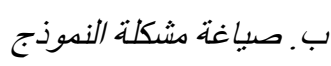

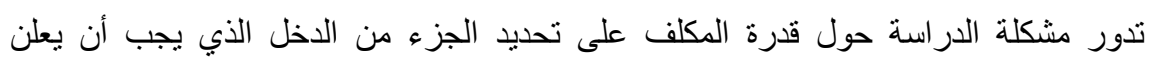

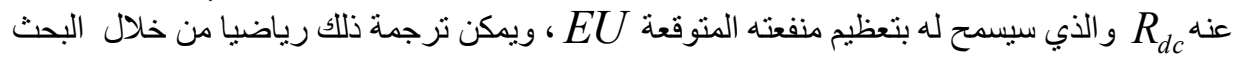

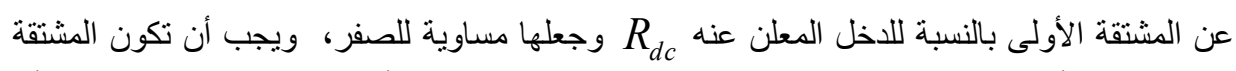

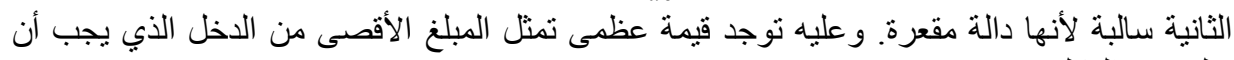
يعلن عنه المكلف.

• نقوم باشتقاق العلاقة [1] بالنسبة للاخل المعلن عنه $R_{d c}$ ونجعلها مساوية للصفر: 


$$
\begin{gathered}
\frac{\partial E U}{\partial R_{d c}}=0 \\
(1-P)(-t) U^{\prime}\left(R_{r}-t R_{d c}\right)+p(-t+s) U^{\prime}\left(R_{r}-t R_{d c}-s\left(R_{r}-R_{d c}\right)\right)=0 \\
-t(1-p) U^{\prime}\left(R_{n d}\right)+p(-t+s) U^{\prime}\left(R_{d}\right)=0 \\
-t(1-p) U^{\prime}\left(R_{n d}\right)-(t-s) p U^{\prime}\left(R_{d}\right)=0
\end{gathered}
$$

• نقوم بانثتقاق العلاقة [2] بالنسبة للاخل المعلن عنه $R_{d c}$ فنحصل على المثتقة الثنانية:

$$
\frac{\partial^{2} E U}{\partial R_{d c}{ }^{2}}=E U^{\prime \prime}
$$

$$
\begin{aligned}
& E U^{\prime \prime}=t^{2}(1-p) U^{\prime \prime}\left(R_{r}-t R_{d c}\right)-(t-s)(-t+s) p U^{\prime \prime}\left(R_{r}+t R_{d c}-s\left(R_{r}-R_{d c}\right)\right) \\
& =t^{2}(1-p) U^{\prime \prime}\left(R_{r}-t R_{d c}\right)-(t-s)(-(t-s)) p U^{\prime \prime}\left(R_{r} \_t R_{d c}-s\left(R_{r}-R_{d c}\right)\right) \\
& =t^{2}(1-p) U^{\prime \prime}\left(R_{r}-t R_{d c}\right)+(t-s)^{2} p U^{\prime \prime}\left(R_{r} \_t R_{d c}-s\left(R_{r}-R_{d c}\right)\right) \\
& E U^{\prime \prime}=D=t^{2}(1-p) U^{\prime \prime}\left(R_{n d}\right)+(t-s)^{2} p U^{\prime \prime}\left(R_{d}\right)[3]
\end{aligned}
$$

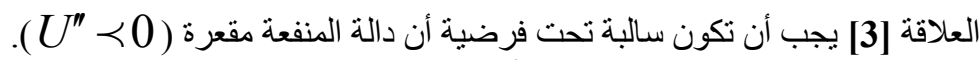

$$
\begin{aligned}
& \text { ثالثا. رصد وتفسير المتغيرات الضريبية للنموذج }
\end{aligned}
$$

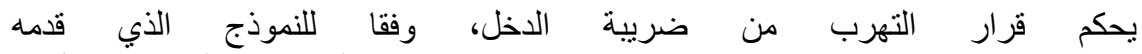

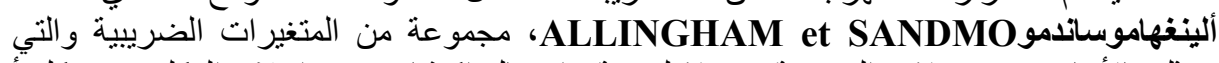

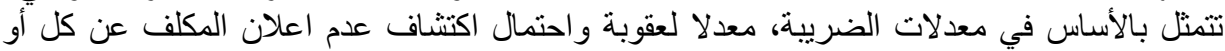

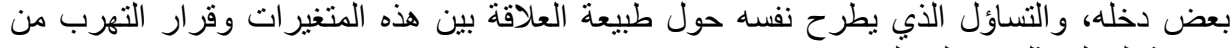

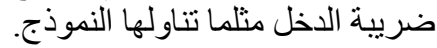


| - معدلات الضريية

يمكن تحليل طبيعة العلاقة بين معدل الضريبة والدخل المعلن عنه من خلال دراسة التغير

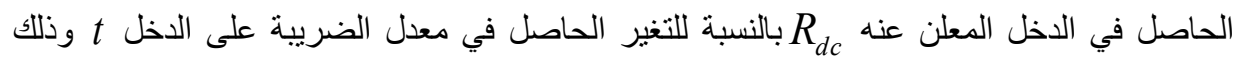

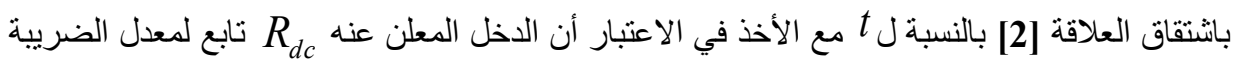

$$
\frac{\partial R_{d c}}{\partial t}=-\frac{R_{d c}\left[t(1-p) U^{\prime \prime}\left(R_{n d}\right)+(t-s) p U^{\prime \prime}\left(R_{d}\right)\right]}{D}+\frac{\left\lfloor(1-p) U^{\prime}\left(R_{n d}\right)+p U^{\prime}\left(R_{d}\right)\right\rfloor}{D}
$$

• باستعمال العلاقة [2] الخاصة بالثرط الأول نتحصل على:

$$
U^{\prime}\left(R_{d}\right)=\frac{-t(1-p)}{(t-s) p} U^{\prime}\left(R_{n d}\right)
$$

• من تعريف مؤشر النفور المطلق نكتب مايلي:

$$
\begin{array}{r}
\operatorname{ding} A\left(R_{n d}\right)=-\frac{U^{\prime \prime}\left(R_{n d}\right)}{U^{\prime}\left(R_{n d}\right)} \\
U^{\prime \prime}\left(R_{n d}\right)=-U^{\prime}\left(R_{n d}\right) A\left(R_{n d}\right)[6] \\
\\
\quad \operatorname{Sig} A\left(R_{d}\right)=-\frac{U^{\prime \prime}\left(R_{d}\right)}{U^{\prime}\left(R_{d}\right)} \\
U^{\prime \prime}\left(R_{d}\right)=-U^{\prime}\left(R_{d}\right) A\left(R_{d}\right)[7]
\end{array}
$$

• بتعويض العلاقات [5]، [6]، [7] في العلاقة [4] نجد:

$$
\begin{gathered}
\frac{\partial R_{d c}}{\partial t}=-\frac{R_{d c}\left[t(1-p)\left(-U^{\prime}\left(R_{n d}\right) A\left(R_{n d}\right)-(t-s) p U^{\prime}\left(R_{d}\right) A\left(R_{d}\right)\right]\right.}{D}+\frac{\left.\mid(1-p) U^{\prime}\left(R_{n d}\right)+p U^{\prime}\left(R_{d}\right)\right]}{D} \\
=-\frac{R_{d c}\left[t(1-p)\left(-U^{\prime}\left(R_{n d}\right) A\left(R_{n d}\right)-(t-s) p \frac{-t(1-p)}{(t-s) p} U^{\prime}\left(R_{n d}\right) A\left(R_{d}\right)\right]\right.}{D}+\frac{\left[(1-p) U^{\prime}\left(R_{n d}\right)+p U^{\prime}\left(R_{d}\right)\right]}{D}
\end{gathered}
$$


$=-\frac{R_{d c}\left[t(1-p)\left(-U^{\prime}\left(R_{n d}\right) A\left(R_{n d}\right)+t(1-p) U^{\prime}\left(R_{n d}\right) A\left(R_{d}\right)\right]\right.}{D}+\frac{\left\lfloor(1-p) U^{\prime}\left(R_{n d}\right)+p U^{\prime}\left(R_{d}\right)\right\rfloor}{D}$

$\frac{\partial R_{d c}}{\partial t}=\frac{R_{d c} t\left[(1-p) U^{\prime}\left(R_{n d}\right)\left(A\left(R_{n d}\right)-A\left(R_{d}\right)\right]\right.}{D}+\frac{\left\lfloor(1-p) U^{\prime}\left(R_{n d}\right)+p U^{\prime}\left(R_{d}\right)\right\rfloor}{D}$

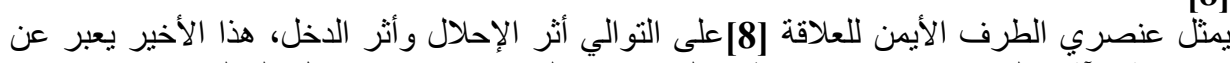

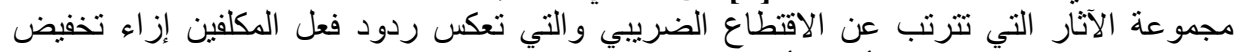

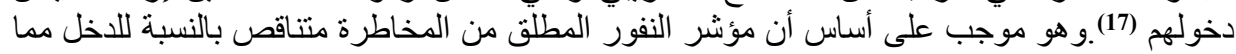

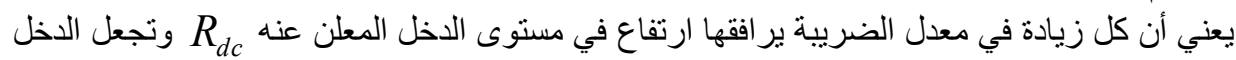

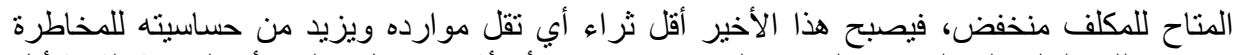

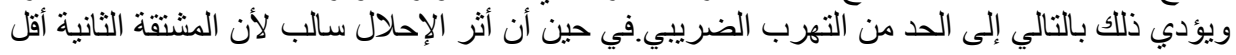

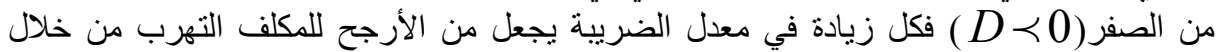
تخفيض مقدار الدخل المعلن عناه. و عليه يقام النموذج أثر إحلال سالب و أثر دخل موجب، وكل من الأثلثرين يعملان في اتجاهين متعاكسين

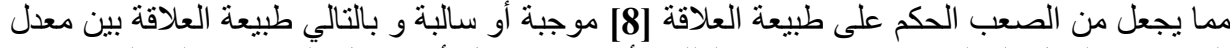
الضريبة والدخل المعلن عنه، وابداء تبعا لذلك رأي قاطع حول أثر معدل الضريبة على على التهرب من

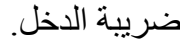

$$
\text { ب بمدل العقوبة }
$$

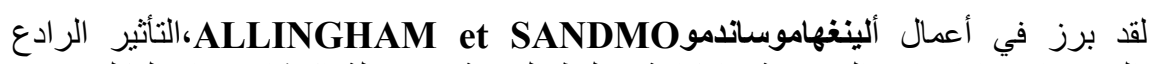

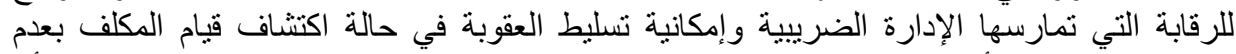

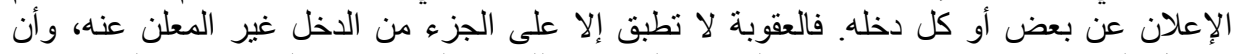

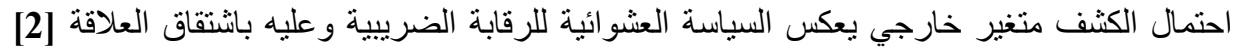

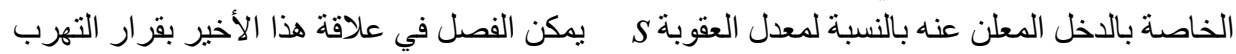

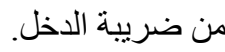

$$
\frac{\partial R_{d c}}{\partial s}=-\frac{\left(R_{r}-R_{d c}\right)(t-s) p U^{\prime \prime}\left(R_{d}\right)}{D}-\frac{p U^{\prime}\left(R_{d}\right)}{D}
$$

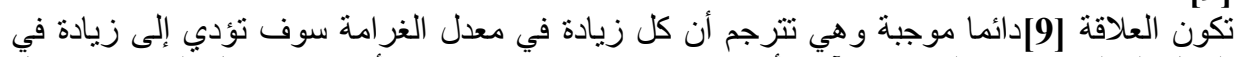

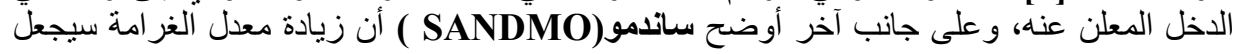
من عرض ساعات العمل في السوق الموازية قليل وبذللك ينخف الدفل الدخل غير المعلن عنه وبالمقابل ترتفع نسبة الدخول المعلن عنهاع (18). 


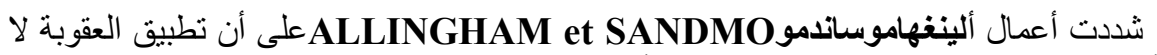

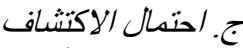

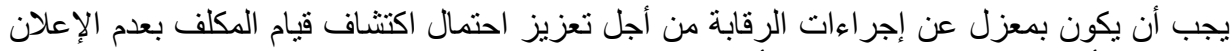

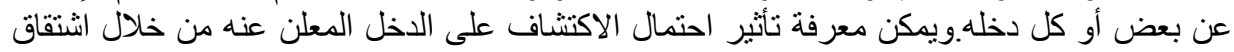

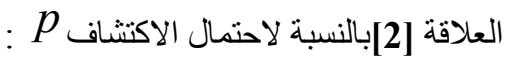

$$
\frac{\partial R_{d c}}{\partial p}=\frac{\left[-t U^{\prime}\left(R_{n d}\right)+(t-s) U^{\prime}\left(R_{d}\right)\right]}{D}
$$

تكون العلاقة [10] موجبة لأن

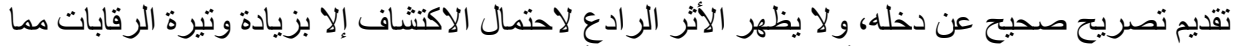

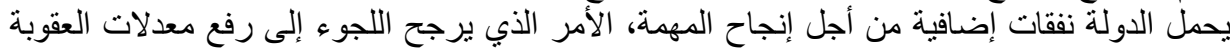
لردع التهرب الضريبي.

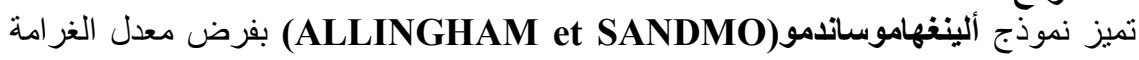

$$
\text { رابعا. نقد النموذج تئز }
$$

على الجزء من الدخل غير المعلن عنه

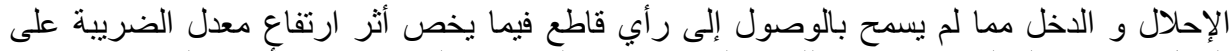

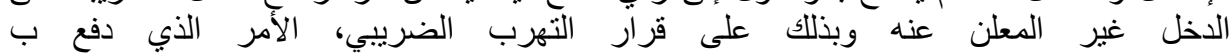
اتزهاكيYITZHAKI منذ عام 1974، إلى دراسة مشكلة التهرب الضريبي في إطار مماتل لذلك الذي لألي حدده ألينغهاموساندموALLINGHAM et SANDMO عام 1972، ولكن تطبق فقط

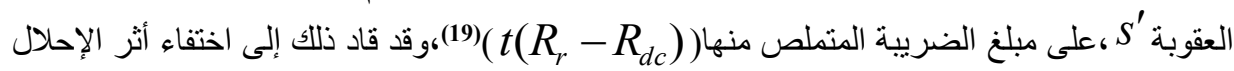

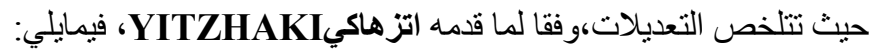

•

$$
\text { ؛ } R_{n d}=R_{r}-t R_{d c}
$$

•

$$
R_{d}=R_{r}-\left(t R_{d c}+s^{\prime} t\left(R_{r}-R_{d c}\right)\right)
$$

• دالة المنفعة المتوقعة للمكلف تكتب بالشكل التالي:

$$
E U=(1-p) U\left(R_{n d}\right)+p U\left(R_{d}\right)
$$




$$
\begin{array}{r}
E U=(1-p) U\left(R_{R}-t R_{d c}\right)+p U\left(R_{r}-R_{d c} t-s^{\prime} t\left(R_{r}-R_{d c}\right)_{[11]}\right. \\
s^{\prime} \succ 1 \text { مع }
\end{array}
$$

وعليه للبحث عن الدخل Rd الذي يجب أن بعلن عنه المكلف والذي يجعل دالة المنفعة الدنوقعة EU

$$
\begin{aligned}
& \frac{\partial E U}{\partial R_{d c}}=E U^{\prime}=0 \\
& (1-p)(-t) U^{\prime}\left(R_{r}-R_{d c} t\right)+p\left(\left(-t+s^{\prime} t\right) U^{\prime}\left(R_{r}-R_{d c} t-s^{\prime} t\left(R_{r}-R_{d c}\right)\right)=0\right. \\
& (1-p)(-t) U^{\prime}\left(R_{r}-R_{d c} t\right)+p\left(t\left(-1+s^{\prime}\right)\right) U^{\prime}\left(R_{r}-R_{d c} t-s^{\prime} t\left(R_{r}-R_{d c}\right)\right)=0 \\
& (t)\left(-(1-p) U^{\prime}\left(R_{n d}\right)+p\left((t)\left(-1+s^{\prime}\right)\right) U^{\prime}\left(R_{d}\right)=0\right. \\
& (t)\left(-(1-p) U^{\prime}\left(R_{n d}\right)+p\left(s^{\prime}-1\right) U^{\prime}\left(R_{d}\right)\right)=0
\end{aligned}
$$

$$
\begin{array}{r}
\frac{\partial^{2} E U}{\partial R_{d c}{ }^{2}}=E U^{\prime \prime}=\bar{D} \prec 0 \\
E U^{\prime \prime}=(t)\left(-(1-p) U^{\prime}\left(R_{r}-R_{d c}\right)+p\left(s^{\prime}-1\right) U^{\prime}\left(R_{r}-R_{d c} t-s^{\prime} t\left(R_{r}-R_{d c}\right)\right)\right) \\
E U^{\prime \prime}=t\left(-(1-p)(-t) U^{\prime \prime}\left(R_{n d}\right)+p\left(s^{\prime}-1\right)\left(-t+s^{\prime} t\right) U^{\prime \prime}\left(R_{d}\right)\right) \\
E U^{\prime \prime}=\bar{D}=t^{2}\left((1-p) U^{\prime \prime}\left(R_{n d}\right)+\left(s^{\prime}-1\right)^{2} p U^{\prime \prime}\left(R_{d}\right)\right)[13]
\end{array}
$$

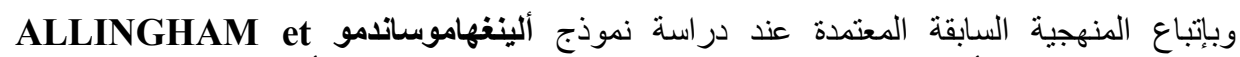

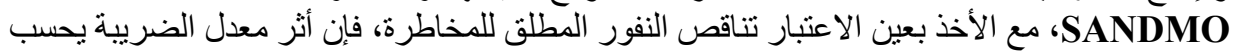
من خلال اشتقاق العلاقة [12]بالنسبة ل $\frac{\partial R_{d c}}{\partial t}=-\frac{t(1-p) U^{\prime}\left(R_{n d}\right)^{\prime}\left\{R_{n d}\left[A\left(R_{d}\right)-A\left(R_{n d}\right)\right]+s^{\prime}\left(R_{r} R_{d c}\right) A\left(R_{d}\right)\right\}}{\bar{D}}$ 


$$
\frac{\partial R_{d c}}{\partial t} \succ 0 \text { وبالاستناد للفرضيات السابقة فابن }
$$

تترجم النتيجة الظاهرة في العلاقة [14] وجود علاقة طردية بين معدل الضريبة ومبلغ الدخل المعلن

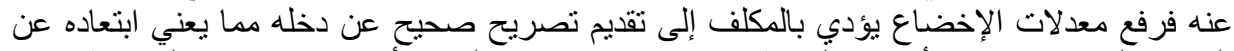

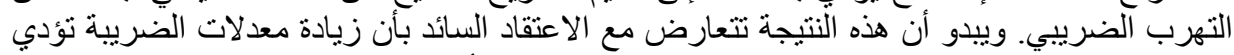

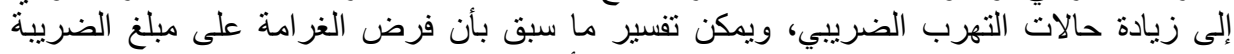

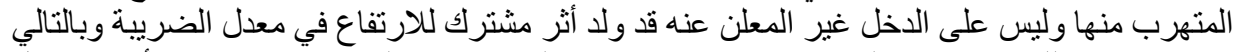

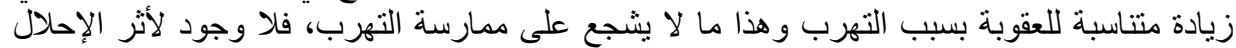

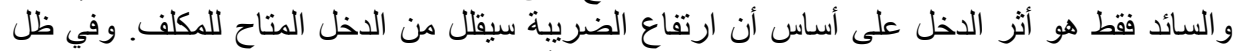

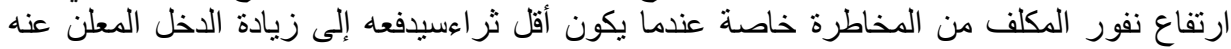

وخفض قيمة التهرب الضريبي.

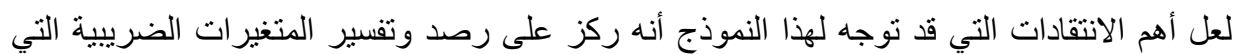

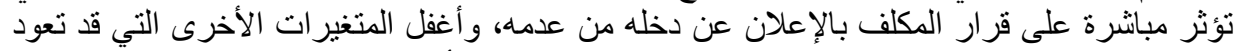

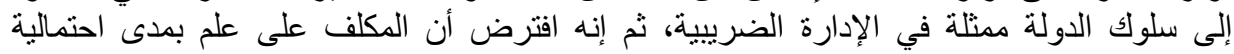

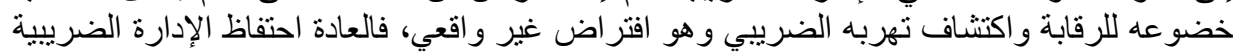
بسرية معلوماتها فيما يخص الرئ الرقابة الضريبية.

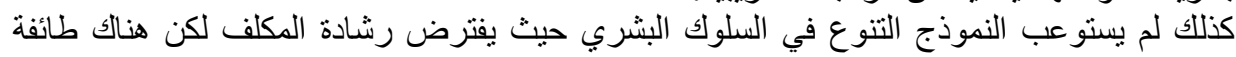

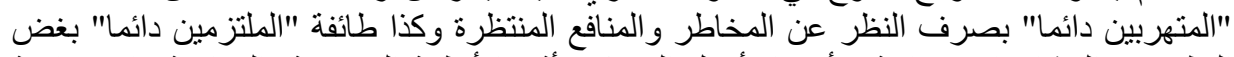

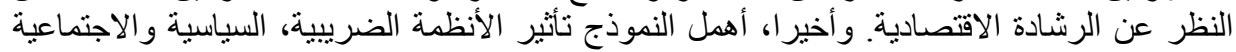

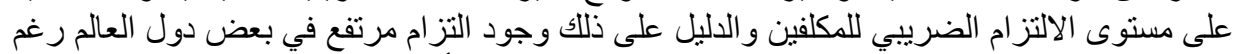

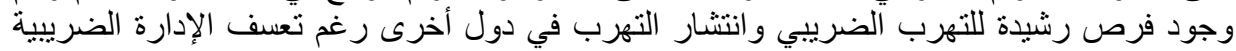

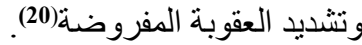

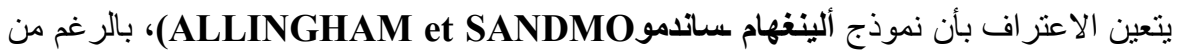

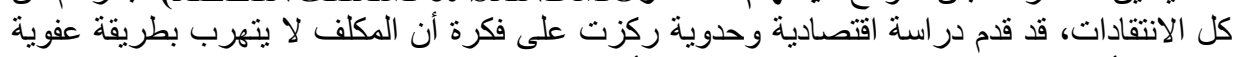

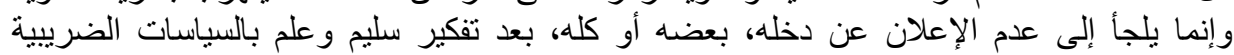

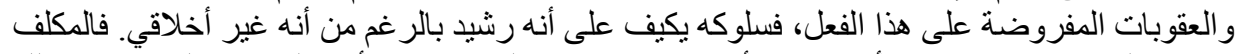

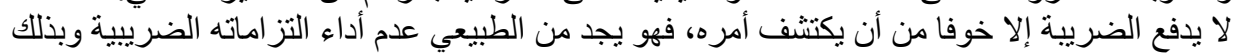

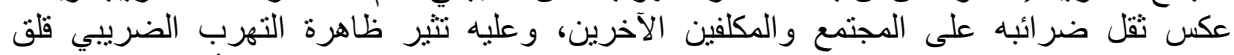

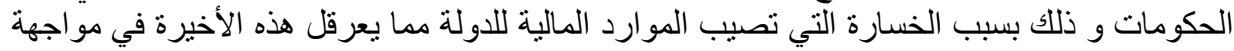

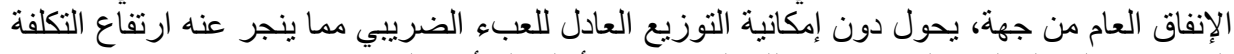

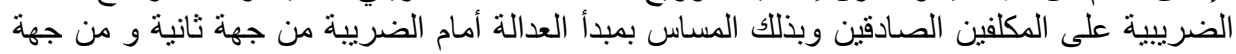
ثالثة فهو يترجم عدم كفاءة الإدارة الضرينية عليبية.

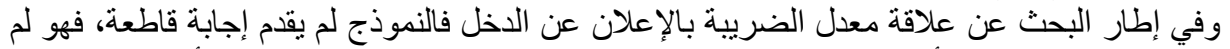

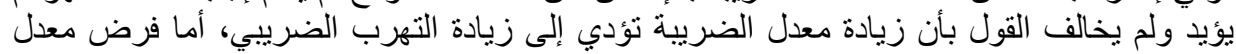

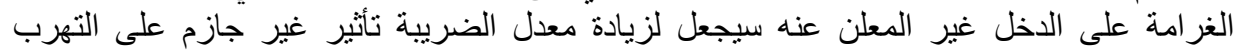

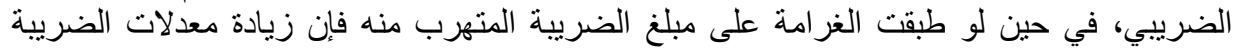
سيؤدي لا محال إلى ارتفاع مستوى الدخل المعلن عنه وبالتالي خفض التهرب التهرب الضريبي. كما يمكناعتبار 
أفضل نسبيا من احتمال الاكتثناف ومعل الغرامة من الأدوات الناجعة لردع التهرب الضريبي غير أن تثنديد الغرامة

(1) ALEXENDRE Jean : Le droit fiscal algérien, OPU, Alger, 1984, P25.

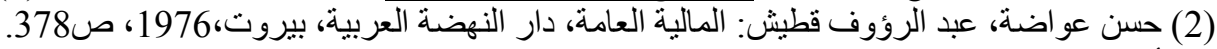

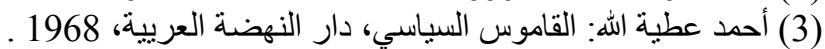

(4) محمد حلمي مراد: تشريع الضرائب، الضرائب العامة، الجزء الضئه الأول، مطبعة النهضة، مصر،

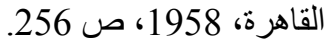

(5) دولاور علي و عبد المنعم فوزي:مالية الدولة،الطبعة الأولى، منشأةالمعارف،الإسكندرية،1962،

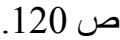

(6) حسن صادق المرصفاوي: التجريم في تثريعات الضرائب، دار المعارف،القاهرة،1963،ص

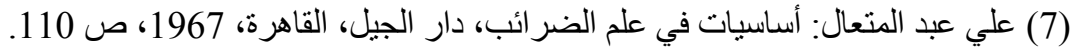

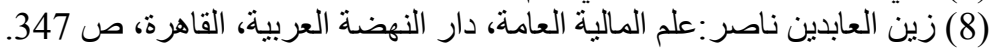

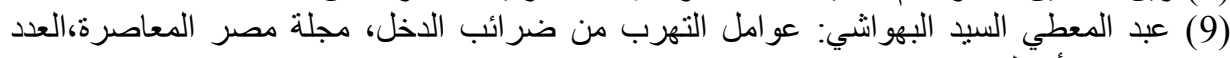

404، مصر ، أفريل 1986، 1984، صبد 41 -42.

(10) عبد الحكيم مصطفى الثرقاوي: التهرب الضريبي والاقتصاد الاسود، دار الجامعة الجديدة،

مصر،2006، صع (10) 111 - 112.

(11) ALLINGHAM. M., SANDMO.A:Incometaxevasion : atheoreticalanalysis, Journal of Public Economics, 1972, PP.323-338.Et Cécile BAZART : La fraude fiscale, modélisation du face à face Etat- contribuable, thèse de doctorat, université monpelier1, France,2000,pp84-88 et p95.

(12) IBID

(13) لقد قدمت هذه النظرية لأول مرة من قبل برنولي (BERNOULLI) سنة 1738، ثم أعيد

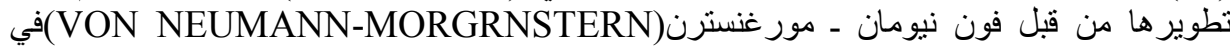

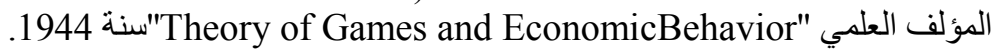
(14) سيد الهواري: الإدارة المالية، الجزء الأول، الاستثمار والتمويل طويل الأجل، دار الجيل للطباعة،

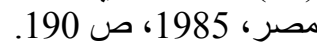

(15) طنيب وعبيدات، محمد شفيق: أساسيات الإدارة المالية في القطاع الخاص، الطبعة الأولى، دار المستقبل، عمان،1997، 1997، ص112.

(16) PRATT.A: Risk aversion in the small and in the large, Economica, Vol.32, 1964, PP.122-136.Et Cécile BAZART, Op.cit, p 85.

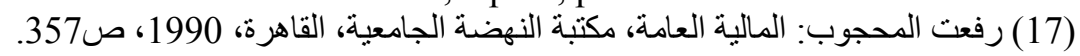

(18) SANDMO.A: Income tax evasion, labour supply and the equity-efficiency tradeoff,

Journal of Public Economics, 1981, PP276.

(19) YITZHAKI.S: A note on income tax evasion, a theoretical analysis, Journal of Public Economics, 1974, PP.201-202.

(20) أحمد جمال الدين موسى: نحو نظرية عامة في التهرب الضريبي، مجلة البحوث القانونية و الاقتصادية، العدد الثامن عشر، جامعة المنصورة، القاهرة، 1995، ص 1 